\title{
Outcomes and management of type A intramural hematoma
}

\author{
Harleen K. Sandhu, Akiko Tanaka, Kristofer M. Charlton-Ouw, Rana O. Afifi, Charles C. Miller III, \\ Hazim J. Safi, Anthony L. Estrera \\ Department of Cardiothoracic and Vascular Surgery, Clinical Science Program, McGovern Medical School at The University of Texas Health \\ Science Center at Houston (UTHealth), Houston, TX, USA \\ Correspondence to: Anthony L. Estrera, MD. Department of Cardiothoracic and Vascular Surgery, McGovern Medical School at UTHealth, 6400 \\ Fannin St., Suite 2850, Houston, TX 77030, USA. Email: Anthony.L.Estrera@uth.tmc.edu.
}

\begin{abstract}
Background: Initial optimal management of acute type A aortic dissection (ATAAD) with intramural hematoma (ATAIMH) remains controversial, especially between centers in the Eastern vs. Western worlds. We examined the literature and our experience to report outcomes after repair of ATAIMH.

Methods: We reviewed the hospital, follow-up clinic records and online mortality databases for all patients who presented to our center for open repair of ATAAD between 1999 and 2014. Preoperative characteristics, early and long-term outcomes were compared between classic ATAAD vs. ATAIMH. Survival was analyzed using Kaplan-Meier and log-rank statistics.

Results: Of the 523 repaired ATAAD, 101 patients (19\%) presented with IMH and 422 (81\%) had classic dissection. ATAIMH were significantly older $(64.8 \pm 12.9$ vs. $56.8 \pm 14.6$ years; $\mathrm{P}<0.001)$, more commonly females (39\% vs. 26\%; $\mathrm{P}=0.010)$, had poor baseline renal function (i.e., glomerular filtration rate) $(\mathrm{P}<0.017)$, more retrograde dissections ( $27 \%$ vs. $8.3 \%$; $\mathrm{P}<0.001)$, and less distal malperfusion $(5 \%$ vs. $15 \% ; \mathrm{P}<0.001)$. Age greater than 60 years, female sex, retrograde dissection, and Marfan syndrome were strongly correlated with ATAIMH. Time to repair for ATAIMH was longer (median, 55.3 vs. 9.8 hours; $\mathrm{P}<0.001$ ) with one death in ATAIMH within three days of presentation $(0.9 \%$ vs. $6 \% ; \mathrm{P}=0.040)$. In all, 30-day mortality in ATAIMH was not different from classic ATAAD (12\% vs.16\%; $\mathrm{P}=0.289)$. A significantly lower incidence of postoperative dialysis in ATAIMH was noted $(10 \%$ vs. $19 \% ; \mathrm{P}=0.034)$. When adjusted for age and renal function, late survival was improved with $\mathrm{IMH}(\mathrm{P}<0.039)$.

Conclusions: ATAIMH continues to be associated with significant morbidity and mortality, comparable to classic aortic dissection. A multidisciplinary management approach involving aggressive medical management and risk stratification for timely surgical intervention, along with genetic profiling, is recommended for optimal care. Long-term monitoring is mandatory to assess compliance to medical therapy and recognition of evolving complications.
\end{abstract}

Keywords: Acute type A dissection; management; intramural hematoma

Submitted Mar 16, 2016. Accepted for publication Jul 04, 2016.

doi: 10.21037/acs.2016.07.06

View this article at: http://dx.doi.org/10.21037/acs.2016.07.06

\section{Introduction}

Acute type A aortic intramural hematomas (ATAIMH) are often included under the spectrum of acute aortic syndromes. This potentially lethal condition is similar to but pathologically distinct from ATAAD and described as "thrombosed-type acute aortic dissection" $(1,2)$. Although both disorders involve hemorrhage into the aortic media, an intimal tear with false lumen is absent in IMH. Although unproven, the pathogenesis for ATAIMH is believed to result from the rupture of vasa vasorum or secondary to a penetrating atherosclerotic ulcer. The risk factor and complications profile is similar to ATAAD as ATAIMH also can result in life-threatening progression along the aortic extent, rupture, hemothorax, tamponade, malperfusion syndromes (cerebral, mesenteric or renal ischemia), and/or 


\begin{tabular}{|c|c|c|}
\hline Major differences & Eastern ${ }^{\# \#}$ & Western ${ }^{\#}$ \\
\hline Incidence (in ATAAD) & $17-40 \%$ & $5-19 \%$ \\
\hline Female & $40-70 \%$ & $30-40 \%$ \\
\hline Age & $54-63$ years & $60-65$ years \\
\hline Initial medical & $50-88 \%$ & $0-49 \%$ \\
\hline Mortality surgery & $9-12 \%$ & $21-33 \%$ \\
\hline Mortality medical & $4-8 \%$ & $33-78 \%$ \\
\hline \multicolumn{3}{|c|}{$\begin{array}{l}\text { \#, based on data from most studies reported from North America } \\
\text { and Europe }(3,5-7) ;{ }^{\# \#} \text {, based on data reported in studies } \\
\text { published by Korean, Japanese, and Chinese investigators (8-10). }\end{array}$} \\
\hline
\end{tabular}

conversion to acute dissection.

The reported incidence of acute aortic IMH in the literature varies between $5-30 \%$ of all acute aortic syndromes $(3,4)$. Most of this variation in reported incidence of ATAIMH is observed in the differential rates reported in the Western and Eastern series. Despite the present gaps in our knowledge of their natural history, popular management approach for ATAIMH, especially in the Western experience (IRAD, Stanford, and other North American authors), continues to be similar to that of ATAAD involving urgent aortic repair (3,5-7). On the other hand, most studies from the Far East recommend initial medical management, with surgery reserved for specific circumstances complicating the course of otherwise stable ATAIMH patients and have reported acceptable early and late outcomes (8-10).

Table 1 highlights some of the major differences in the literature reporting experience in management of ATAIMH from the Western $v s$. Eastern centers.

Similar conclusions on the observed heterogeneity in IMH incidence and presentation were made by other authors who examined the literature $(11,12)$. Optimal management of ATAIMH remains controversial given the conflicting reports in the literature, which vary from initial medical management with expectant surgery $(10,13)$ to aggressive treatment by either open or endovascular approach for ATAIMH $(11,14)$ In our experience, despite timely medical management, conversion of ATAIMH to typical dissection presented a significant risk beyond eight days (15). We previously reported our experience with management of ATAIMH comparing the outcomes of various management approaches involving immediate surgical or delayed (expectant for up to three days) repair, and medical management. However, this intentional delay did not prove to be especially advantageous (16).

Given the paucity of data on patient characteristics or factors affecting risk for rupture or mortality, and a lack of consensus on appropriate strategy for ATAIMH management, we examined our large, single-center experience involving a uniform management protocol to determine baseline and treatment-related predictors correlated with presentation and outcomes in ATAIMH. We placed a special focus on examining the potential characteristics correlated with an increased propensity of IMH diagnosed at presentation. Furthermore, we analyzed the predictors of early and long-term mortality for risk stratification for effective triage of these high-risk patients by prioritizing timely management of those in need of immediate surgical repair.

\section{Methods}

\section{Patients and study design}

This study was designed as a retrospective cohort study and was approved by the institutional review board (IRB), the Committee for the Protection of Human Subjects at McGovern Medical School at UTHealth and Memorial Hermann Hospital in Houston, Texas. Consent was waived for the retrospective analysis. All patients included in the repaired ATAAD database between October 1999 and December 2014 were reviewed.

\section{Definitions}

Acute type A IMH (ATAIMH) was defined as previously described (16), an acute ascending aortic dissection without identifiable intimal tear and an absence of flow in the false lumen. All ATAAD patients underwent a computed tomography (CT) scan on admission. Radiological evidence for identification of intimal flap comes from admission CT and the presence of a dissection flap is confirmed by transesophageal echocardiography. Detailed defining criteria for patients included as ATAIMH have been described elsewhere $(15,16)$. Aortic dissection (and/or $\mathrm{IMH}$ ) was defined as acute if the time of the initial onset of pain (or related symptoms, in hours) was determined to be within two weeks of presentation. Time to surgery was defined as time from presentation to the operative repair of ATAAD. Typical or classic dissection referred to aortic dissection with evidence (radiological or 
intraoperative) of free flow into the false channel. Early mortality refers to any death occurring within 30 days of surgery. The baseline renal function or status was determined by the admission glomerular filtration rate (GFR) calculated by the Cockcroft-Gault method (17). Pulmonary complications included respiratory dysfunction (defined as prolonged intubation $>24$ hours, reintubation, or need for tracheostomy), pleural effusion, pneumothorax, pneumonia, acute respiratory distress syndrome, and pulmonary embolism following aortic repair. Reoperation was defined as the need for any surgical procedure involving reopening the previous incision, redo procedure in the same segment of aorta including re-exploration for bleeding, an abdominal procedure (such as exploratory laparotomy) or an aorto/vascular procedure. A systolic blood pressure $<90 \mathrm{~mm} \mathrm{Hg}$ was regarded as hypotension. Aortic insufficiency was defined as "significant" in cases with moderate to severe grade, and aortic rupture was defined as clinical/intraoperative confirmation presence of blood in the pericardial sac with tamponade or observation of frank/ active bleeding, with or without preoperative radiological evidence. Malperfusion syndrome included any clinically evident vascular bed perfusion deficit, including cerebral, spinal, visceral, renal, coronary, and limb.

\section{Surgical management}

We have previously described our detailed surgical approach for classic acute ascending aortic dissection $(15,16)$. Briefly, all cases of classic ATAAD undergo an urgent or emergent repair, excluding devastating neurologic injury, metastatic cancer, or patient refusal. For ATAIMH patients, operative repair is performed emergently in those who present in extremis, while surgery is usually delayed (in anticipation of later surgery) in those who are hemodynamically stable on admission. Our surgical technique includes utilization of cardiopulmonary bypass (CPB), deep hypothermic circulatory arrest, and retrograde cerebral perfusion, as described elsewhere $(16,18)$. Cardiopulmonary bypass is frequently initiated via femoral or axillary artery cannulation with axillary artery cannulation used only in cases with poor femoral artery pulses. Adequacy of arterial perfusion is routinely confirmed by cerebral oximetry and corresponding decrease in nasopharyngeal and bladder temperatures. Venous cannulation is performed via the superior and inferior vena cava. Both antegrade and retrograde cold blood cardioplegia are used for myocardial protection. A left ventricular sump is inserted into the right superior pulmonary vein. Upon achieving the target cooling temperatures, cardiopulmonary bypass is discontinued, the circulation is arrested, and retrograde cerebral perfusion is started via the superior vena cava cannula. After placing the patient in a slight Trendelenburg position, the ascending aorta is then opened and inspected. In patients with classic ATAAD, the intimal tear is resected upon identification. Concomitant aortic arch replacement is performed for cases with enlargement $>5 \mathrm{~cm}$, while a partial arch replacement (hemiarch) with an open distal anastomosis is performed in a majority of the cases. Careful inspection of the aortic wall is performed to remove any remaining thrombus prior to performing the anastomosis. Upon completion of the anastomosis and de-airing of the aortic graft, a clamp is placed on the graft and antegrade perfusion is initiated via a sidearm branch of the aortic graft. Following the initiation of systemic warming, the proximal reconstruction involving the remainder of the ascending aorta is resected to the sinotubular junction. The aortic valve is carefully examined and, if intact, a proximal anastomosis is performed after resuspension of the aortic valve in the absence of aortic root enlargement $(>5 \mathrm{~cm})$ or compromise.

\section{Statistical analysis}

Analysis was performed in two separate stages. First, we assessed the clinical correlates of a radiological or intraoperative confirmation of IMH, in order to better define this group of patients and assess the propensity for occurrence of IMH and associated outcomes. We then examined the independent and joint effects of IMH presentation and other risk factors on short- and long-term survival. For univariate analysis, discrete variables were analyzed by contingency table methods and continuous variables were compared between groups using $t$-tests or Wilcoxon rank-sum statistics. Adjusted analyses were conducted by multivariable statistics using multiple logistic regressions (MLR).

For short-term outcomes, stratified analyses were conducted following univariate statistics to assess potential confounding or joint effects of the risk factors on mortality. Multivariable analyses for predictors of short-term outcomes were conducted using MLR and additional attributable-risk estimates were computed using generalized linear models (GLM). These models present adjusted percent risk for calculated outcome that can be attributable to the risk factor. Long-term survival, stratified by IMH, was assessed using Kaplan-Meier analysis and Cox 
proportional hazards regression. The hazard for mortality represents the instantaneous probability of death for each life table interval. All computations were performed using SAS software version 9.4 (SAS Institute Inc., Cary, NC, USA).

\section{Results}

\section{Demographics}

We repaired 523 ATAADs. Of these, 101 (19\%) patients presented with IMH and $81 \%$ had classic ATAAD. The patients with ATAIMH were significantly older $(64.8 \pm 12.9$ vs. $56.8 \pm 14.6$ years; $\mathrm{P}<0.001)$ and predominantly females (39\% vs. $26 \% ; \mathrm{P}=0.010$ ). ATAIMH patients presented with a reduced renal function compared to the classic ATAAD ( $\mathrm{P}=0.017)$; less frequently with shortness of breath, abdominal pain, malperfusion syndromes, leg ischemia, significant aortic insufficiency, and retrograde dissection (Tables 1,2).

Overall, median time from admission to surgery was 11.3 (IQR, 3-31.5) hours for the whole cohort, and was significantly longer (as expected) in ATAIMH 55.3 (IQR, 8.8-105.3) hours as opposed to 9.8 (IQR, 2.5-19) hours for typical ATAAD. Aortic diameter, rate of rupture, branch vessel involvement, or coronary dissection were not different between the two groups (Table 3). Cannulation strategy, extent of arch repair, aortic root replacement, and blood product use was not significantly different between the ATAIMH and classic ATAAD groups (Table 3). In the ATAIMH group, all operative times were considerably shorter, including cardiopulmonary bypass time, aortic cross-clamp time, and retrograde cerebral perfusion times when compared with the typical ATAAD (Table 3).

\section{Correlates of ATAIMH}

Screening the univariate risk factors (Tables 2,3) and preliminary Spearman correlation analysis revealed several baseline patient characteristics and presenting features that were significantly associated with occurrence of IMH in ATAAD. Additional multivariable analysis on all of these factors through step-wise MLR model selection and followup manual variable selection was performed to construct the final model of determinants correlated with increased propensity of IMH in ATAAD (Table 4). The key correlates included age over 65 years, female gender, Marfan syndrome, and retrograde dissection.

\section{Perioperative outcomes}

Overall, there was no significant difference in incidence of postoperative complications, namely strokes, spinal or paraplegic neurological complications, visceral malperfusion, hemodynamic, and respiratory impairment (Table 5). Postoperative renal dysfunction requiring dialysis was lower in ATAIMH compared to typical ATAAD (10\% vs. $19 \%$, respectively; $\mathrm{P}=0.034$ ).

Overall 30-day mortality after aortic repair was $15.3 \%$ (80/523) for the total cohort and there was no significant difference seen between ATAIMH and typical ATAAD patients $12 \%(12 / 101)$ vs. $16 \%(68 / 422 ; \mathrm{P}=0.289)$. After controlling for time to surgery, risk of early mortality was significantly lower among those who underwent aortic repair on the second or third day following admission between ATAIMH vs. ATAAD (0/21 vs. 7/37; relative risk in ATAAD $=1.2 ; 95 \% \mathrm{CI}, 1.1-1.4 ; \mathrm{P}=0.034)$. The hazard or instantaneous risk for mortality was not statistically different between ATAIMH vs. classic ATAAD (Figure 1; $\mathrm{P}=0.204)$. Furthermore, an increase in hazard of mortality among ATAIMH patients was observed at around 6 days, with another peak around 18 days after presentation.

\section{Long-term survival outcomes}

Over a median follow-up of 3.8 (IQR, 0.6-8.2) years, the unadjusted 10 -year survival after ATAAD was not significantly different from ATAIMH (55.1\% vs. 60.3\%; $\mathrm{P}=0.204)$. Poor baseline renal function [hazard ratio $(\mathrm{HR})=1.5 ; \mathrm{P}=0.04$; Figure $2 A$ ] and older age $(>65$ years; $\mathrm{HR}=2.7 ; \mathrm{P}<0.001)$ significantly affected long-term survival and was significantly lower in typical ATAAD when compared to ATAIMH (Figure 2B). Conversely, the unadjusted hazard of long-term mortality associated with classic ATAAD $(\mathrm{HR}=1.2, \mathrm{P}<0.213)$ was not statistically different from ATAIMH. However, the difference in effect of ATAIMH vs. ATAAD on mortality became apparent and pronounced following adjustment for age and baseline renal function (significantly increasing ATAAD HR to 1.6 following adjustment; $\mathrm{P}=0.039$; Figure 3). Long-term survival at 1-, 5- and 10-years for ATAIMH was $85.8 \%, 81.1 \%$, and $66.7 \%$, respectively, and was significantly better than survival of classic ATAAD: $78.8 \%, 72.4 \%$, and $54.3 \%$, respectively $(\mathrm{P}<0.039)$, adjusted for age and renal function.

\section{Risk-factor and multivariate analysis}

Multivariable analysis on all the significant variables 


\begin{tabular}{|c|c|c|c|}
\hline Variable & ATAIMH n=101 (19.3\%) & ATAAD $n=422(80.7 \%)$ & $P$ value \\
\hline \multicolumn{4}{|l|}{ Patient characteristics } \\
\hline Females & $39(38.6)$ & $109(25.8)$ & 0.010 \\
\hline Males & $62(61.4)$ & $313(74.2)$ & 0.010 \\
\hline Age & $65(56-76)$ & $56(46-67.9)$ & $<0.001$ \\
\hline \multicolumn{4}{|l|}{ Comorbid conditions } \\
\hline HTN & $85(84.2)$ & $329(77.9)$ & 0.169 \\
\hline COPD & $27(26.7)$ & $101(23.9)$ & 0.557 \\
\hline PVD & $13(12.9)$ & $31(7.4)$ & 0.072 \\
\hline Smoking & $56(55.5)$ & $224(53.1)$ & 0.669 \\
\hline Substance use & $9(8.9)$ & $29(6.9)$ & 0.478 \\
\hline CAD & $15(14.9)$ & $84(19.9)$ & 0.244 \\
\hline Previous MI & $6(5.9)$ & $18(4.3)$ & 0.435 \\
\hline Previous CAB/PCI & $7(6.9)$ & $42(9.9)$ & 0.349 \\
\hline Prior sternotomy & $4(3.9)$ & $42(9.9)$ & 0.056 \\
\hline Connective/genetic & $5(4.9)$ & $16(3.8)$ & 0.576 \\
\hline Marfan syndrome & $3(2.9)$ & $8(1.9)$ & 0.452 \\
\hline BAV & $3(1.9)$ & $36(8.5)$ & 0.056 \\
\hline Previous h/o dissection & $16(15.8)$ & $24(5.7)$ & $<0.001$ \\
\hline Previous thoracic procedure & $2(1.9)$ & $19(4.5)$ & 0.396 \\
\hline Previous AA procedure & $6(5.9)$ & $19(4.5)$ & 0.602 \\
\hline \multicolumn{4}{|l|}{ Presentation } \\
\hline Chest pain & $81(80.2)$ & $335(79.4)$ & 0.855 \\
\hline Shortness of breath & $8(7.9)$ & $90(21.3)$ & 0.002 \\
\hline Back pain & $51(50.1)$ & $176(41.7)$ & 0.109 \\
\hline Abdominal pain & $8(7.9)$ & $63(14.9)$ & 0.065 \\
\hline Syncope & $15(14.9)$ & $36(8.5)$ & 0.054 \\
\hline Hypotension & $15(14.9)$ & $92(21.8)$ & 0.119 \\
\hline Aortic insufficiency & $49(48.5)$ & $296(70.1)$ & $<0.001$ \\
\hline Cardiac arrest & $6(5.9)$ & $12(2.8)$ & 0.132 \\
\hline MPS & $24(23.8)$ & $144(34.1)$ & 0.045 \\
\hline Lower limb ischemia/MPS & $5(4.9)$ & $64(15.2)$ & 0.006 \\
\hline Lower limb weakness & $5(4.9)$ & $22(5.2)$ & 0.915 \\
\hline Stroke on admission & $5(4.9)$ & $33(7.8)$ & 0.318 \\
\hline${ }^{*}$ eGFR $(n=466 ; 91 ; 375)^{\#}$ & $79.5 \pm 35.2$ & $90.1 \pm 46.4$ & 0.017 \\
\hline GFR Q1 ( $\leq 58)$ & $28(30.8)$ & $88(23.5)$ & 0.148 \\
\hline GFR Q2 (>58 to $\leq 81.9)$ & $25(27.5)$ & $92(24.5)$ & 0.562 \\
\hline GFR Q3 (>81.9 to $\leq 106.3)$ & $21(23.1)$ & $97(25.9)$ & 0.583 \\
\hline GFR Q4 (>106.3) & $17(18.7)$ & $98(26.1)$ & 0.139 \\
\hline
\end{tabular}

*, eGFR= Cockcroft-Gault estimated GFR mL/min $1.73 \mathrm{~m}^{2} .{ }^{*}$, missing data; $(n=x x x ; x x ; x x x)$ represents total patients in cohort; ATAIMH; ATAAD with available data. Categorical data shown as number(\%) and continuous data as mean \pm standard-deviation or median (Interquartile range or IQR). AA, abdominal aortic; BAV, bicuspid aortic valve; CAB, coronary artery bypass; CAD, coronary artery disease; COPD, chronic obstructive pulmonary disease; GFR, glomerular filtration rate; HTN, hypertension; MI, myocardial infarction; MPS, malperfusion syndrome; $\mathrm{PCl}$, percutaneous coronary intervention; PVD, peripheral vascular disease; Connective/genetic, connectivetissue disorder or hereditary/genetic disease. 


\begin{tabular}{|c|c|c|c|}
\hline Variable & ATAIMH n=101 (19.3\%) & ATAAD $n=422(80.7 \%)$ & $P$ value \\
\hline Adm-rep (hrs) $(n=487 ; 99 ; 388)^{\#}$ & $55.3(8.8-105.3)$ & $9.8(2.5-19.1)$ & $<0.001$ \\
\hline Retrograde type A & $27(26.7)$ & $35(8.3)$ & $<0.001$ \\
\hline Rupture & $20(19.8)$ & $71(16.8)$ & 0.478 \\
\hline Femoral & 78 (79.6) & $341(83.9)$ & 0.297 \\
\hline Ascending aorta & $17(17.4)$ & $50(12.3)$ & 0.188 \\
\hline Axillary & $3(3.1)$ & $15(3.7)$ & 1.000 \\
\hline \multicolumn{4}{|l|}{ Operative repair } \\
\hline Aortic valve resuspension & $87(86.1)$ & $355(84.1)$ & 0.615 \\
\hline Aortic root reconstruction & $81(80.2)$ & $323(76.5)$ & 0.431 \\
\hline Coronary bypass grafting & $5(4.9)$ & $35(8.3)$ & 0.256 \\
\hline Naso temp $\left({ }^{\circ} \mathrm{C}\right)(\mathrm{n}=511 ; 100 ; 411)$ & $17.9 \pm 2.1$ & $17.9 \pm 2.7$ & 0.895 \\
\hline Bypass time (min) $(n=521 ; 100 ; 421)$ & $152.6 \pm 51.2$ & $164.3 \pm 52.7$ & 0.046 \\
\hline Aortic clamp (min) $(n=521 ; 100 ; 421)$ & $92.6 \pm 35.1$ & $103.3 \pm 33.0$ & 0.004 \\
\hline Circ arrest (min) $(n=518 ; 101 ; 417)$ & $26.7 \pm 11.4$ & $29.5 \pm 11.0$ & 0.025 \\
\hline ACP & $3(2.9)$ & $9(2.1)$ & 0.709 \\
\hline Platelets $(n=520 ; 100 ; 420)^{\#}$ & ${ }^{*} 0=33,12[6-12]$ & ${ }^{*} 0=112,12[6-12]$ & 0.112 \\
\hline Cryo $(n=520 ; 100 ; 420)^{\#}$ & ${ }^{*} 0=79,10[10-20]$ & ${ }^{*} 0=279,10[10-20]$ & 0.171 \\
\hline
\end{tabular}

\#, Missing data; $(\mathrm{n}=\mathrm{xxx} ; \mathrm{xx} ; \mathrm{xxx}$ ) represents total patients in cohort; ATAIMH; ATAAD with available data. Categorical data shown as number(\%) and continuous data as mean \pm standard-deviation or median (Interquartile range or IQR). *, Blood products are zeroinflated continuous variables. $0=x x$ - indicates number of patients who received 0 units of blood product. The presented data is after adjusting for zero values. Adm-rep, admission to repair time; Asc, ascending aorta; cm, centimeters; circ arrest, circulatory arrest time; Cryo, cryoprecipitates; hrs, hours; IQR, interquartile range; IMH, intramural hematoma; FFP, fresh frozen plasma; Nasotemp, lowest nasopharyngeal temperature; PRBC, packed red blood cells; RCP, retrograde cerebral perfusion; min, minutes.

obtained from univariate analysis, revealed significant risk for early mortality associated with age $>65$ years, poor baseline renal function, rupture, and $\mathrm{CAD}(\mathrm{OR}=4.9 ; 95 \%$ CI, 2.7-8.9, $\mathrm{P}<0.001)$ (Table 4). Further quantification of mortality-risk using GLM-analysis demonstrated that nearly $9.4 \%$ of early mortalities in ATAAD could be attributed to rupture alone and that IMH had no independent effect on risk for mortality (Tables 6,7).

Long-term survival was significantly associated with older age (HR, 2.63/year; $\mathrm{P}<0.0001)$, baseline GFR $(\mathrm{HR}=0.99 /$ unit increase in GFR; $\mathrm{P}<0.029)$, presence of IMH $(\mathrm{HR}=0.61 ; \mathrm{P}<0.028)$, Marfan syndrome $(\mathrm{HR}=7.3$; 


\begin{tabular}{|c|c|c|c|}
\hline Variable & ATAIMH n=101 (19.3\%) & ATAAD n=422(80.7\%) & $P$ value \\
\hline Reoperation for bleeding & $9(8.9)$ & $66(15.6)$ & 0.083 \\
\hline Stroke & $13(12.9)$ & $31(7.4)$ & 0.072 \\
\hline Neurological complications & $32(31.7)$ & $116(27.5)$ & 0.401 \\
\hline Ml & $1(0.9)$ & $4(0.9)$ & 0.969 \\
\hline Cardiac arrest & $10(9.9)$ & $47(11.1)$ & 0.720 \\
\hline Gastrointestinal complications & $26(25.7)$ & $126(29.9)$ & 0.413 \\
\hline Dialysis ${ }^{\star *}$ & $10(9.9)$ & $79(18.7)$ & 0.034 \\
\hline 30-day mortality ${ }^{\ddagger}$ & $12(11.9)$ & $68(16.1)$ & 0.289 \\
\hline ICU LOS (days) (n=471;93;378) & $5[3-10]$ & $6[3-13]$ & 0.447 \\
\hline LOS (days) $(n=505 ; 99 ; 406)^{\#}$ & $14[10-20]$ & $13[9-19]$ & 0.301 \\
\hline
\end{tabular}

${ }^{* *}$, patients on dialysis preoperatively were not evaluated for post-op dialysis; *, missing data; $(n=x x x ; x x ; ~ x x x)$ represents total patients in cohort; ATAIMH, ATAAD with available data. Categorical data shown as number (\%) and continuous data as mean \pm standard-deviation or median (Interquartile range or IQR). ${ }^{\dagger}$, death within 24-hours of aortic repair; ${ }^{\ddagger}$, death within 30 -days of aortic repair; AMS, altered mental status; ICU, intensive care unit; LOS, length of stay; MI, myocardial infarction.

Table 5 Correlates of IMH on admission in ATAAD

\begin{tabular}{|c|c|c|c|c|c|}
\hline Variable & Parameter estimate & Odds ratio & $95 \%$ confidence limits & Attributable risk ${ }^{\star *}$ & $P$ value \\
\hline Age $>60$ years & 1.0353 & 2.82 & $1.72-4.60$ & $14.3 \% \pm 3.4 \%$ & $<0.001$ \\
\hline Female & 0.4927 & 1.64 & $1.01-2.65$ & $7.4 \% \pm 3.7 \%$ & 0.045 \\
\hline Marfan & 1.3557 & 3.88 & $0.96-15.67$ & $19.1 \% \pm 11.6 \%$ & 0.057 \\
\hline Retrograde dissection & 1.5894 & 4.90 & $2.71-8.87$ & $12.7 \% \pm 5.1 \%$ & $<0.001$ \\
\hline
\end{tabular}

${ }^{*}$, odds-ratios, parameter estimates, $95 \%$ confidence-intervals and P value from MLR; ${ }^{\star \star}, \%$ attributable mortality $( \pm$ Standard error) obtained from GLM-analysis.

$\mathrm{P}<0.001)$, CAD $(\mathrm{HR}=1.88 ; \mathrm{P}<0.001)$, significant $\mathrm{PVD}[4.3$ (0.15)], and rupture (HR=1.7; $\mathrm{P}=0.028)$ in multiple $\mathrm{Cox}$ regression. Tables 6,7 lists other important determinants of long-term survival.

\section{Discussion}

It has been consistently observed that ATAIMH patients are older and more frequently female than those with classic $\operatorname{ATAAD}(4,10,11,16)$. In addition to older age and female gender, other historical and presenting characteristics were markedly different in ATAIMH patients, as previously discussed and listed in Table 2. They had poor baseline renal function, previous distal or retrograde dissection, and a lower incidence of shortness of breath, abdominal pain, aortic insufficiency, syncope, lower limb ischemia, and malperfusion syndromes on admission. Therefore, the importance of better defining these patients at the time of admission, especially given the controversy surrounding the timing of aortic repair in ATAIMH, prompted us to analyze 


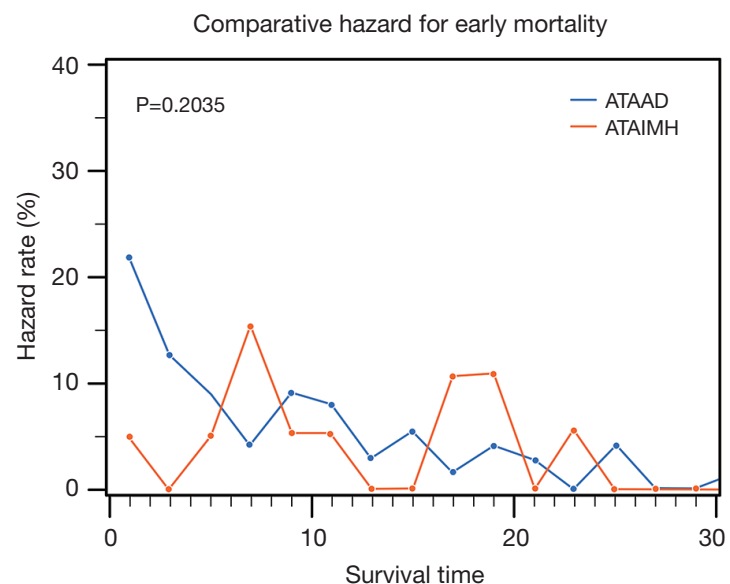

Figure 1 Hazard for early mortality among ATAIMH compared to typical aortic dissection.

A

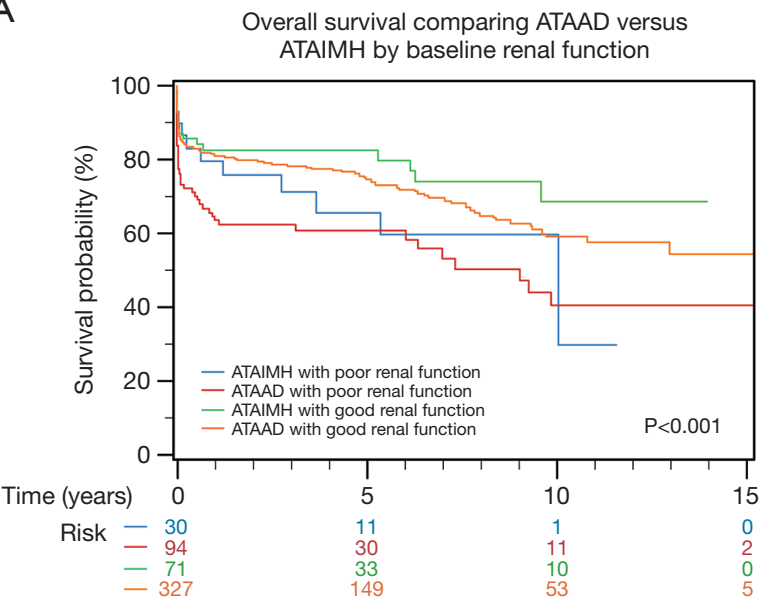

B

Overall survival comparing ATAAD versus ATAIMH by age

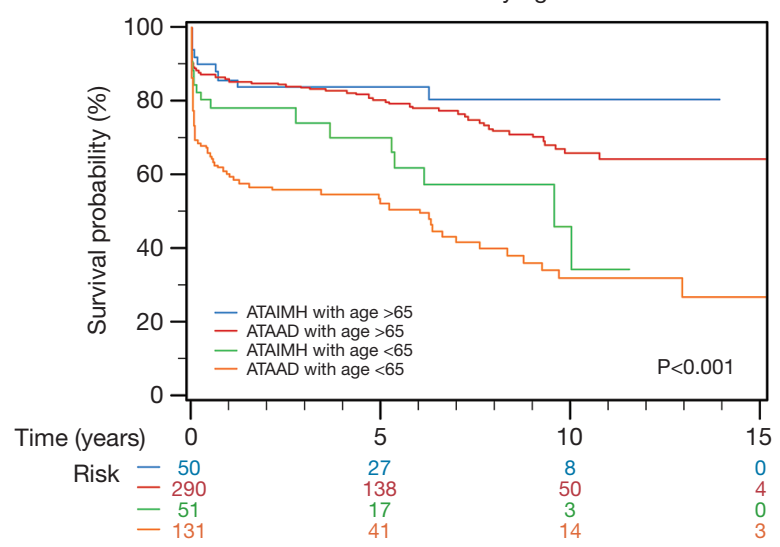

Figure 2 Long-term survival for acute type A aortic IMH vs. acute type A aortic dissections.

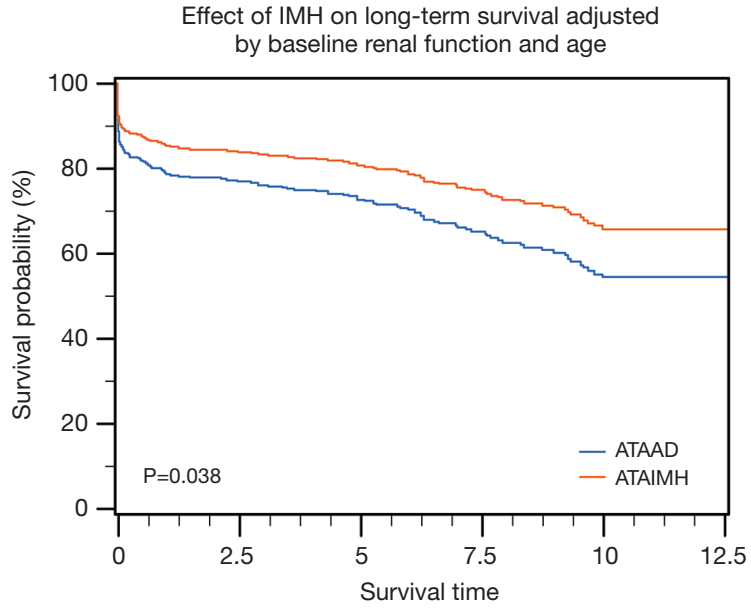

Figure 3 Effect of baseline renal function and age on long-term survival.

the clinical correlates of IMH in acute dissection. Age over 65 years was associated with 2.5 -fold (95\% CI, 1.5-3.9), female gender with 1.6-fold (95\% CI, 1-2.7), Marfan syndrome with 3.2-fold (95\% CI, 0.8-12.7), and retrograde dissection with 4.9-fold (95\% CI, 2.7-8.9) increased odds of ATAIMH.

ATAIMH cases typically underwent "timely" aortic repair within an average time of 55-hours from admission and only a small proportion underwent an emergent ( $<12$-hours from admission) surgery, owing to complicated or unstable status (such as malperfusion syndrome or rupture), when compared to typical ATAAD (30\% vs. 58\%, respectively). When looking at the risk of early mortality and influence of IMH, there was no major difference was observed in ATAIMH vs. ATAAD (12\% vs. 16\%; $\mathrm{P}=0.289)$. When we closely examined the risk of early death attributable to timing of surgery, we noted that $50 \%$ of early deaths in ATAIMH cases occurred in patients who underwent aortic repair within 24 hours owing to complicated presentation, with the remainder of the $50 \%$ mortality seen in those who had an aortic repair more than three days after admission. It was noteworthy that no early mortality occurred in ATAIMH patients who had a "timely" repair deferred up to 48-72 hours after admission.

Furthermore, death rate within 72 hours of admission was significantly lower in ATAIMH vs. ATAAD [1/101 (0.9\%) vs. 25/422 (5.9\%); $\mathrm{P}=0.04]$. However, the single death seen in ATAIMH occurred in a patient who required surgery within 12 hours of admission. When controlling for time to surgery, no additional risk for early mortality 


\begin{tabular}{|c|c|c|c|c|}
\hline Variable & $\mathrm{OR}^{*}$ & 95\% confidence limits & Attributable risk ${ }^{\star *}$ & $P$ value ${ }^{\star \star}$ \\
\hline Age $\geq 65$ years & 1.84 & 0.99-3.39 & $7.7 \% \pm 3.7 \%$ & 0.038 \\
\hline CAD & 1.99 & $1.09-3.66$ & $9.2 \% \pm 3.9 \%$ & 0.019 \\
\hline Rupture & 1.91 & $1.01-3.60$ & $9.4 \% \pm 4.2 \%$ & 0.026 \\
\hline
\end{tabular}

\begin{tabular}{lllll}
\multicolumn{2}{l}{ Table 7 Adjusted effects of risk factors on long-term survival } & & \\
\hline Variable & HR $^{\#}$ & $95 \%$ confidence limits & \\
\hline Age $\geq 65$ years & 2.950 & $2.04-4.26$ & Parameter estimate $^{\#}$ & P value $^{\#}$ \\
\hline Marfan & 6.072 & $2.52-14.66$ & 1.08181 & $<0.0001$ \\
Baseline GFR & 0.994 & $0.98-0.99$ & 1.80375 & 0.0001 \\
CAD & 1.786 & $1.25-2.55$ & -0.00619 & 0.0179 \\
Prior PV bypass & 4.679 & $1.430-15.3$ & 0.58001 & 0.0015 \\
\hline Hypotension & 1.934 & $1.344-2.78$ & 1.54318 & 0.0107 \\
IMH & 0.635 & $0.407-0.99$ & 0.65950 & 0.0004 \\
\hline
\end{tabular}

\#, estimates from Cox proportional-hazards regression analysis. CAD, coronary artery disease; GFR, baseline glomerular filtration rate; HR, hazard ratio; MPS, malperfusion syndrome; IMH, intramural hematoma.

was noted following emergent, urgent, or delayed aortic repair, that could be associated with ATAIMH over and above that observed in classic dissection. However, risk of early mortality was significantly lower among those who underwent aortic repair on the second or third day following admission in ATAIMH vs. ATAAD (0/21 vs. 7/37, respectively; RR in ATAAD $=1.2$; 95\% CI, 1.1-1.4; $\mathrm{P}=0.034)$. These findings supported our previous hypothesis for "timely" intervention in ATAIMH $(15,16)$ that deferring surgery in an otherwise non-emergent indication for ATAAD could be advantageous as it allows time for aortic wall thickening and stabilization of the intimal flap, thereby facilitating aortic repair and lower operative mortality.

Extrapolating from prior experience with other acute aortic pathologies, including ruptured ATAAD and ruptured abdominal aortic aneurysm $(19,20)$, it was evident that risk stratification in this patient group was essential for prioritization and management planning for patients in need of immediate repair, as opposed to those who could benefit from a deferred intervention. Therefore, we performed the risk factor analysis for early mortality, which revealed that in addition to age $\geq 65$ years, renal function, and $\mathrm{CAD}$, rupture was a significant predictor of early mortality (Tables 6,7). Propensity for ATAIMH or occurrence of IMH had no independent effect on early mortality. However, age and renal status adjusted longterm survival was significantly improved in ATAIMH compared to classic ATAAD (Figure 3).

\section{Limitations}

As a retrospective analysis, the results of this study should be viewed with inherent limitations and biases. As with any observational study, the presented results should be limited to hypothesis derivation only. We acknowledge that assessment of the precise effect of intentional delay in aortic intervention is limited, due to the exclusion of patients who were medically managed or who died prior to surgery from the comparison group. Since the current database is structured to include all "repaired" ATAAD patients, this limitation is a drawback of the current database design. Long-term follow up on those who were managed medically compared to these repaired patients is also not known at this time. All characterizations were made 
based on clinical or intraoperative observations with limited radiological assessments, especially during the early part of our experience and for long-term postoperative assessment among those who were lost to follow up.

We are working on improving the current database to include these patients and hope to analyze these results in the near future. This might impact our understanding and current results on risk factor profile for mortality in association with ATAIMH. Hence, for the present study, we have restricted the interpretations of our analysis on risk factors to correlational inferences between the significant determinants and a presentation of ATAIMH.

\section{Conclusions}

IMH in patients with acute type A dissection continues to be associated with significant morbidity and mortality that is comparable to classic aortic dissection. The current results show favorable operative outcomes with the institutional approach of deferred (beyond 72 hours) intervention for $\mathrm{IMH}$ in all but unstable patients, and those which are comparable to the outcomes after emergent surgery in ATAAD. These findings stand to reason that "timely" intervention (within the first 3 days) can prove beneficial to reduce operative mortality for stable ATAIMH. Multidisciplinary management approach involving aggressive medical management and risk stratification for timely surgical intervention, along with genetic profiling, is recommended for optimal care. Long-term monitoring is mandatory to assess compliance to medical therapy and recognition of evolving complications. Further studies to evaluate the determinants and correlates of ATAIMH are required in order to aid timely risk-stratification of this high-risk patient population.

\section{Acknowledgements}

None.

\section{Footnote}

Conflicts of Interest: The authors have no conflicts of interest to declare.

Ethical Statement: This study was approved by the institutional review board (IRB), the Committee for the Protection of Human Subjects at McGovern Medical School at UTHealth and Memorial Hermann Hospital in
Houston, Texas.

\section{References}

1. Uchida K, Imoto K, Karube N, et al. Intramural haematoma should be referred to as thrombosed-type aortic dissection. Eur J Cardiothorac Surg 2013;44:366-9; discussion 369.

2. Krukemberg E. Beiträge zur Frage des Aneurysma dissecans. Beitr Pathol Anat Allg Pathol 1920;67:329-51.

3. Evangelista A, Mukherjee D, Mehta RH, et al. Acute intramural hematoma of the aorta: a mystery in evolution. Circulation 2005;111:1063-70.

4. Sundt TM. Intramural hematoma and penetrating atherosclerotic ulcer of the aorta. Ann Thorac Surg 2007;83:S835-41; discussion S846-50.

5. Robbins RC, McManus RP, Mitchell RS, et al. Management of patients with intramural hematoma of the thoracic aorta. Circulation 1993;88:II1-10.

6. Roselli EE, Svensson LG. Aggressive surgical repair for ascending intramural hematoma is still a great option. J Thorac Cardiovasc Surg 2016;151:359-60.

7. Svensson LG, Adams DH, Bonow RO, et al. Aortic valve and ascending aorta guidelines for management and quality measures: executive summary. Ann Thorac Surg 2013;95:1491-505.

8. Kitai T, Kaji S, Yamamuro A, et al. Clinical outcomes of medical therapy and timely operation in initially diagnosed type a aortic intramural hematoma: a 20 -year experience. Circulation 2009;120:S292-8.

9. Matsushita A, Fukui T, Tabata M, et al. Preoperative characteristics and surgical outcomes of acute intramural hematoma involving the ascending aorta: A propensity score-matched analysis. J Thorac Cardiovasc Surg 2016;151:351-8.

10. Moizumi Y, Komatsu T, Motoyoshi N, et al. Clinical features and long-term outcome of type A and type B intramural hematoma of the aorta. J Thorac Cardiovasc Surg 2004;127:421-7.

11. Chou AS, Ziganshin BA, Charilaou P, et al. Longterm behavior of aortic intramural hematomas and penetrating ulcers. J Thorac Cardiovasc Surg 2016;151:361-72, 373.e1.

12. Pelzel JM, Braverman AC, Hirsch AT, et al. International heterogeneity in diagnostic frequency and clinical outcomes of ascending aortic intramural hematoma. J Am Soc Echocardiogr 2007;20:1260-8.

13. Song JK, Yim JH, Ahn JM, et al. Outcomes of patients 
with acute type a aortic intramural hematoma. Circulation 2009; 120:2046-52.

14. Song JK. Aortic intramural hematoma: aspects of pathogenesis 2011. Herz 2011;36:488-97.

15. Estrera A, Miller C 3rd, Lee TY, et al. Acute type A intramural hematoma: analysis of current management strategy. Circulation 2009;120:S287-91.

16. Estrera AL, Sandhu HK, Leake SS, et al. Early and late outcomes of acute type A aortic dissection with intramural hematoma. J Thorac Cardiovasc Surg 2015;149:137-42.

17. Cockcroft DW, Gault MH. Prediction of creatinine

Cite this article as: Sandhu HK, Tanaka A, CharltonOuw KM, Afifi RO, Miller CC 3rd, Safi HJ, Estrera AL. Outcomes and management of type A intramural hematoma. Ann Cardiothorac Surg 2016;5(4):317-327. doi: 10.21037/ acs.2016.07.06 clearance from serum creatinine. Nephron 1976;16:31-41.

18. Safi HJ, Miller CC 3rd, Lee TY, et al. Repair of ascending and transverse aortic arch. J Thorac Cardiovasc Surg 2011;142:630-3.

19. Afifi RO, Sandhu HK, Leake SS, et al. Determinants of Operative Mortality in Patients With Ruptured Acute Type A Aortic Dissection. Ann Thorac Surg 2016;101:64-71.

20. Azizzadeh A, Miller CC 3rd, Villa MA, et al. Effect of patient transfer on outcomes after open repair of ruptured abdominal aortic aneurysms. Vascular 2009;17:9-14. 